\title{
$\rho$ meson decay width from 2+1 flavor lattice QCD
}

\section{N. Ishizuka ${ }^{* a, b \dagger}$ for PACS-CS Collaboration,}

${ }^{a}$ Graduate School of Pure and Applied Sciences, University of Tsukuba, Tsukuba 305-8571,

Japan

${ }^{b}$ Center for Computational Sciences, University of Tsukuba, Tsukuba 305-8577, Japan

We perform a lattice QCD study of the $\rho$ meson decay from the $N_{f}=2+1$ full QCD configurations generated with a renormalization group improved gauge action and a non-perturbatively $O(a)$-improved Wilson fermion action. The resonance parameters, the effective $\rho \rightarrow \pi \pi$ coupling constant and the resonance mass, are estimated from the $P$-wave scattering phase shift for the isospin $I=1$ two-pion system. The finite size formulas are employed to calculate the phase shift from the energy on the lattice. Our calculations are carried out at two quark masses, $m_{\pi}=410 \mathrm{MeV}\left(m_{\pi} / m_{\rho}=0.46\right)$ and $m_{\pi}=300 \mathrm{MeV}\left(m_{\pi} / m_{\rho}=0.35\right)$, on a $32^{3} \times 64(L a=2.9 \mathrm{fm})$ lattice at the lattice spacing $a=0.091 \mathrm{fm}$. We compare our results at these two quark masses with those given in the previous works using $N_{f}=2$ full QCD configurations and the experiment.

XXIX International Symposium on Lattice Field Theory - Lattice 2011

July 10-16, 2011

Squaw Valley, Lake Tahoe, California

\footnotetext{
*Speaker.

${ }^{\dagger}$ E-mail : ishizuka@ccs.tsukuba.ac.jp
} 


\section{Introduction}

Study of the $\rho$ meson decay is a significant step for understanding the dynamical aspects of hadron interactions with lattice QCD. In the early stage of studies toward this direction the transition amplitude $\langle\pi \pi \mid \rho\rangle$ extracted from the time behavior of the correlation function $\langle\pi(t) \pi(t) \rho(0)\rangle$ was used to estimate the decay width, assuming that the hadron interaction is small $[1,2,3,4]$.

A more realistic approach is a study from the $P$-wave scattering phase shift for the isospin $I=1$ two-pion system. The finite size formulas presented by Lüscher in the center of mass frame [5] and extensions to non-zero total momentum frames $[6,7]$ are employed for an estimation of the phase shift from an eigenvalue of the energy on the lattice. The first study of this approach was carried out by CP-PACS Collaboration using $N_{f}=2$ full QCD configurations $\left(m_{\pi}=330 \mathrm{MeV}, a=0.21 \mathrm{fm}\right.$, $L a=2.5 \mathrm{fm}$ ) [8]. After this work ETMC Collaboration presented results with $N_{f}=2$ configurations at several quark masses $\left(m_{\pi}=290,330 \mathrm{MeV}(L a=2.5 \mathrm{fm}), m_{\pi}=420,480 \mathrm{MeV}(L a=1.9 \mathrm{fm})\right.$, $a=0.079 \mathrm{fm})[9,10]$. Recently Lang et al. reported results of high statistical calculations on a single $N_{f}=2$ gauge ensemble ( $m_{\pi}=266 \mathrm{MeV}, a=0.124 \mathrm{fm}, L a=1.98 \mathrm{fm}$ ) [11].

In the present work we extend these studies by employing $N_{f}=2+1$ full QCD configurations and working on a larger lattice volume. Our calculations are carried out with the gauge configurations previously generated by PACS-CS Collaboration with a renormalization group improved gauge action and a non-perturbatively $O(a)$-improved Wilson fermion action at $\beta=1.9$ on $32^{3} \times 64$ lattice ( $a=0.091 \mathrm{fm}, L a=2.9 \mathrm{fm}$ ) [12]. We choose two subsets of the PACS-CS configurations. One of them corresponds to the hopping parameters $\kappa_{u d}=0.13754$ for the degenerate up and down quarks and $\kappa_{s}=0.13640$ for the strange quark, for which the pion mass takes $m_{\pi}=410 \mathrm{MeV}$ $\left(m_{\pi} / m_{\rho}=0.46\right)$. The other is at $\kappa_{u d}=0.13770$ and $\kappa_{s}=0.13640$, corresponding to $m_{\pi}=300 \mathrm{MeV}$ $\left(m_{\pi} / m_{\rho}=0.35\right)$. All calculations are carried out on the PACS-CS computer at Center for Computational Sciences, University of Tsukuba. Details of our calculations are presented in Ref. [13].

We note that QCDSF Collaboration calculated the scattering phase shifts for the ground state in the center of mass frame at several quark masses. $\left(m_{\pi}=240-430 \mathrm{MeV}\right)$ [14]. They estimated the resonance parameters from these results, assuming that the effective $\rho \rightarrow \pi \pi$ coupling constant does not depend on the quark mass. BMW Collaboration presented their first preliminary results with $N_{f}=2+1$ configurations $\left(m_{\pi}=200,340 \mathrm{MeV}, a=0.116 \mathrm{fm}\right)$ at Lattice 2010 [15].

\section{Method}

In order to calculate the $P$-wave scattering phase shift for the isospin $I=1$ two-pion system at various energies from a single full QCD ensemble, we consider three momentum frames, the center of mass frame (CMF), the non-zero momentum frames with total momentum $\mathbf{P}=(2 \pi / L)(0,0,1)$ (MF1) and $\mathbf{P}=(2 \pi / L)(1,1,0)(\mathrm{MF} 2)$, as carried out in the previous works by ETMC $[9,10]$ and Lang et al. [11]. In the present work we calculate the scattering phase shifts for four irreducible representations : $\mathbf{T}_{1}^{-}$in the CMF, $\mathbf{A}_{2}^{-}$and $\mathbf{E}^{-}$in the MF1, and $\mathbf{B}_{1}^{-}$in the MF2. The finite size formulas for these representations are given in Refs. [5, 6, 7].

For the $\mathbf{T}_{1}^{-}$and the $\mathbf{E}^{-}$representation, we only calculate the scattering phase for the ground state in the present work. The energy of the ground state is much smaller than that of the excited state on our gauge configurations. Thus the energy of these states can be extracted by a single 
exponential fit for the time correlation functions of the $\rho$ meson. We use the local $\rho$ meson operator for the sink and a smeared operator for the source as discussed later.

For the $\mathbf{A}_{2}^{-}$and the $\mathbf{B}_{1}^{-}$representation, we also calculate the scattering phase shift for the first excited state. In order to extract the energies of the lowest two state for these representations, we use the variational method [17] with a matrix of the time correlation function,

$$
G(t)=\left(\begin{array}{ll}
\left\langle 0\left|(\pi \pi)^{\dagger}(t) \overline{(\pi \pi)}\left(t_{s}\right)\right| 0\right\rangle & \left\langle 0\left|(\pi \pi)^{\dagger}(t) \bar{\rho}\left(t_{s}\right)\right| 0\right\rangle \\
\left\langle 0\left|\rho^{\dagger}(t) \overline{(\pi \pi)}\left(t_{s}\right)\right| 0\right\rangle & \left\langle 0\left|\rho^{\dagger}(t) \bar{\rho}\left(t_{s}\right)\right| 0\right\rangle
\end{array}\right),
$$

for each representation. The energies are extracted from two eigenvalues $\lambda_{n}(t)(n=1,2)$ of the matrix $M(t)=G(t) G^{-1}\left(t_{R}\right)$ with some reference time $t_{R}$, assuming that the lower two states dominate the correlation function.

In (2.1) the operator $\rho(t)$ is given by $\rho(t)=\sum_{j=1}^{3} p_{j} \cdot \rho_{j}(\mathbf{p}, t) /|\mathbf{p}|$, where $\rho_{j}(\mathbf{p}, t)$ is the local operator for the neutral $\rho$ meson at the time slice $t$ with the momentum $\mathbf{p}$. The momentum takes $\mathbf{p}=$ $(2 \pi / L)(0,0,1)$ for the $\mathbf{A}_{2}^{-}$and $\mathbf{p}=(2 \pi / L)(1,1,0)$ for the $\mathbf{B}_{1}^{-}$representation. Hereafter we assume that the momentum $\mathbf{p}$ takes one of these two values depending on the representation. $(\pi \pi)(t)$ is an operator for the two pions with the momentum $\mathbf{0}$ and $\mathbf{p}$, which is defined by

$$
(\pi \pi)(t)=\frac{1}{\sqrt{2}}\left(\pi^{+}\left(\mathbf{0}, t_{1}\right) \pi^{-}(\mathbf{p}, t)-\pi^{-}\left(\mathbf{0}, t_{1}\right) \pi^{+}(\mathbf{p}, t)\right) \times \mathrm{e}^{m_{\pi} \cdot\left(t_{1}-t\right)},
$$

where $\pi^{ \pm}(\mathbf{p}, t)$ is the local pion operator with the momentum $\mathbf{p}$ at the time slice $t$. The time slice of the pion with the zero momentum is fixed at $t_{1} \gg t$, and the time slice of the other pion $t$ runs over the whole time extent. An exponential time factor in (2.2) is introduced so that the operator has the same time behavior as that of the usual Heisenberg operator, i.e., $\langle 0|(\pi \pi)^{\dagger}(t)=\langle 0|(\pi \pi)^{\dagger}(0) \mathrm{e}^{-H t}$, with the Hamiltonian $H$.

Two operators $\overline{(\pi \pi)}\left(t_{s}\right)$ and $\bar{\rho}\left(t_{s}\right)$ are used for the sources in (2.1), which are given by

$$
\begin{aligned}
& \overline{(\pi \pi)}\left(t_{s}\right)=\frac{1}{\sqrt{2}}\left(\pi^{+}\left(\mathbf{0}, t_{s}\right) \pi^{-}\left(\mathbf{p}, t_{s}\right)-\pi^{-}\left(\mathbf{0}, t_{s}\right) \pi^{+}\left(\mathbf{p}, t_{s}\right)\right), \\
& \bar{\rho}\left(t_{s}\right)=\frac{1}{N_{\Gamma}} \sum_{\mathbf{z} \in \Gamma} \frac{1}{\sqrt{2}}\left(\bar{U}\left(\mathbf{z}, t_{s}\right) \gamma_{p} U\left(\mathbf{z}, t_{s}\right)-\bar{D}\left(\mathbf{z}, t_{s}\right) \gamma_{p} D\left(\mathbf{z}, t_{s}\right)\right) \mathrm{e}^{i \mathbf{p} \cdot \mathbf{z}},
\end{aligned}
$$

where $\gamma_{p}=\sum_{j=1}^{3} p_{j} \cdot \gamma_{j} /|\mathbf{p}|$. The operator $Q\left(\mathbf{z}, t_{s}\right)(Q=U, D)$ is a smeared operator for the up or the down quark given by $Q\left(\mathbf{z}, t_{s}\right)=\sum_{\mathbf{x}} q\left(\mathbf{x}, t_{s}\right) \cdot \Psi(|\mathbf{x}-\mathbf{z}|)$, where $q\left(\mathbf{x}, t_{s}\right)(q=u, d)$ is the up or the down quark at the position $\mathbf{x}$ and the time $t_{s}$. We adopt the same smearing function $\Psi(|\mathbf{x}|)$ as in Ref. [12]. This operator is used after fixing gauge configurations to the Coulomb gauge. In (2.4) a summation over $\mathbf{z}$ is taken to reduce a statistical error and $\Gamma=\left\{\mathbf{z} \mid \mathbf{z}=(L / 2) \cdot\left(n_{1}, n_{2}, n_{3}\right), n_{j}=\right.$ 0 or $\left.1, N_{\Gamma}=8\right\}$ is chosen in the present work. The smeared operator (2.4) is also used to extract the energy of the ground state for the $\mathbf{T}_{1}^{-}$and the $\mathbf{E}^{-}$representation, setting the momentum $\mathbf{p}=\mathbf{0}$ and $\mathbf{p}=(2 \pi / L)(0,0,1)$, respectively.

The periodic boundary conditions are imposed for both spatial and temporal directions in configuration generations. We impose the Dirichlet boundary condition for the temporal direction in calculations of the quark propagators, to avoid the unwanted thermal contributions produced by propagating two pions in opposite directions in a time. For both quark masses, we set the source 

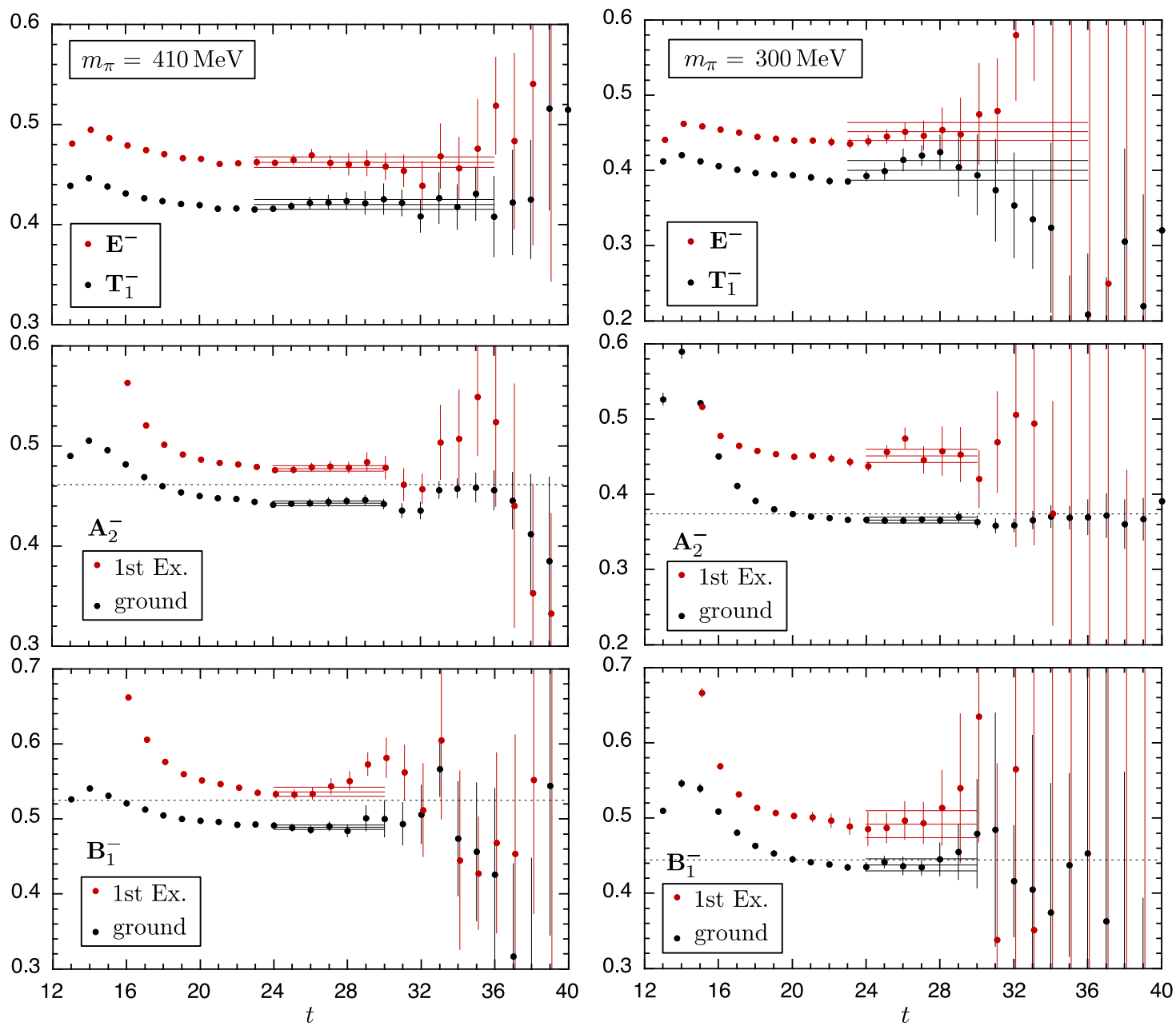

Figure 1: Effective masses for six states considered in the present work at $m_{\pi}=410 \mathrm{MeV}$ (left panel) and $m_{\pi}=300 \mathrm{MeV}$ (right panel).

operators at $t_{s}=12$ to avoid effects from the temporal boundary, and the zero momentum pion in the sink operator $(\pi \pi)(t)$ in (2.2) at $t_{1}=42$. The total number of configurations at $m_{\pi}=410 \mathrm{MeV}$ is 440 and that $m_{\pi}=300 \mathrm{MeV}$ is 400 . We calculate the quark contractions of $G(t)$ in (2.1) by the source method and the stochastic noise method as in the previous work by CP-PACS [8]. Details of the method of the calculations are explained in Ref. [13].

\section{Results}

The effective masses of the time correlation functions for six states considered in the present work are plotted in Fig. 1, where we choose $t_{R}=23$ as the reference time of the variational method. We can find plateaus in the time region $t \geq 23$. We extract the energy by a single exponential fit for the time correlation functions. In Fig. 1 the results of the fitting with one standard deviation error band are also expressed by solid lines. The dotted line for the $\mathbf{A}_{2}^{-}$and $\mathbf{B}_{1}^{-}$representation in the figure indicates the energy of the two free pions for each representation. 

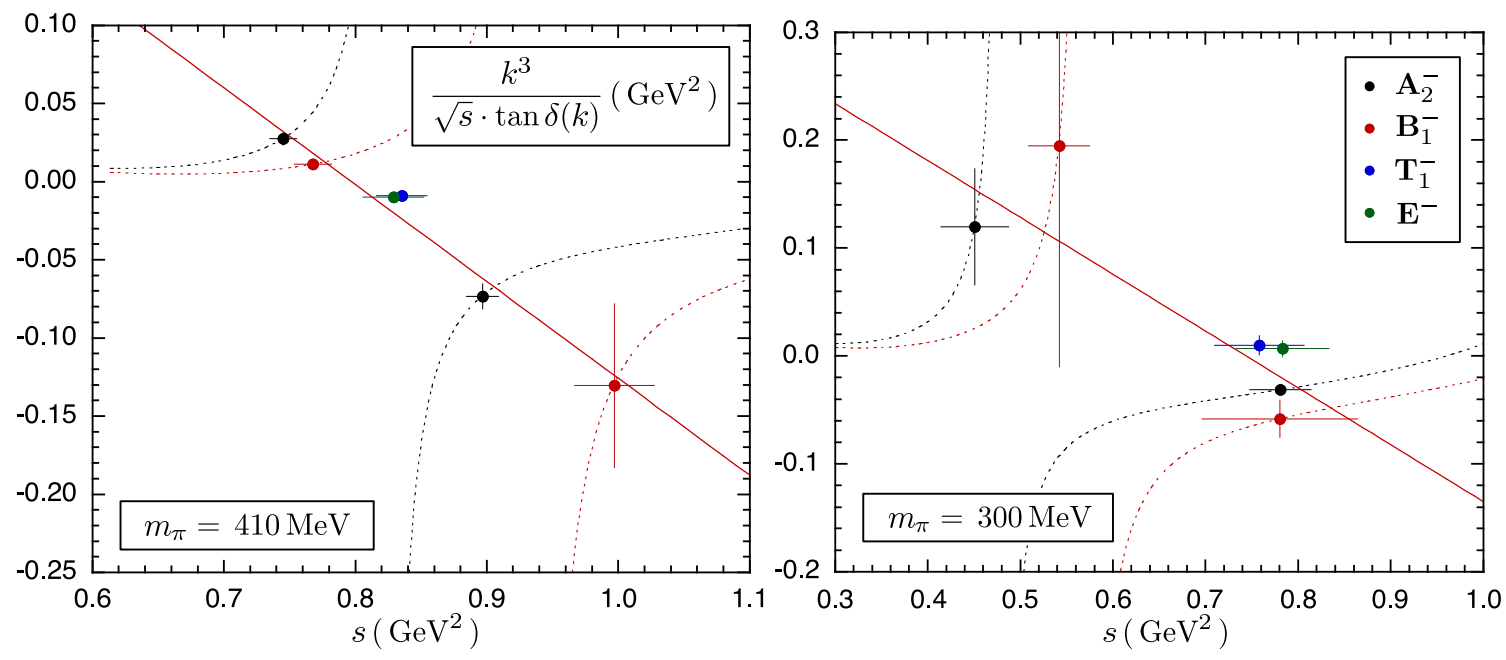

Figure 2: $\left(k^{3} / \tan \delta(k)\right) / \sqrt{s}$ as a function of square of the invariant mass $s$ at $m_{\pi}=410 \mathrm{MeV}$ (left panel) and $m_{\pi}=300 \mathrm{MeV}$ (right panel).

Converting the energies for each representation to the invariant masses $\sqrt{s}$ and substituting them into the finite size formulas, we obtain the scattering phase shifts plotted in Fig. 2. In the figure we show $\left(k^{3} / \tan \delta(k)\right) / \sqrt{s}$ as a function of square of the invariant mass $s$, where $k=\sqrt{s / 4-m_{\pi}^{2}}$ is the scattering momentum. The finite size formulas for the $\mathbf{A}_{2}^{-}$and the $\mathbf{B}_{1}^{-}$representation are plotted by dotted lines.

In order to extract the resonance parameters from our results of the scattering phase shift, we parametrize the phase shift with the effective $\rho \rightarrow \pi \pi$ coupling constant $g_{\rho \pi \pi}$ and the the resonance mass $m_{\rho}$ by

$$
\frac{k^{3}}{\tan \delta(k)} / \sqrt{s}=\frac{6 \pi}{g_{\rho \pi \pi}^{2}}\left(m_{\rho}^{2}-s\right) .
$$

This parametrization has been widely used in the previous works of the $\rho$ meson decay. By chisquare fitting of the scattering phase shifts with the fit function (3.1), we obtain,

$$
\begin{aligned}
& g_{\rho \pi \pi}=5.52 \pm 0.40 \quad, \quad m_{\rho}=892.8 \pm 5.5 \pm 13 \mathrm{MeV} \quad \text { at } m_{\pi}=410 \mathrm{MeV} \\
& g_{\rho \pi \pi}=5.98 \pm 0.56 \quad, \quad m_{\rho}=863 \pm 23 \pm 12 \mathrm{MeV} \quad \text { at } m_{\pi}=300 \mathrm{MeV} \text {, }
\end{aligned}
$$

where the second error of $m_{\rho}$ is the systematic uncertainty for the determination of the lattice spacing. In Fig. 2 we draw the fitting curves by solid red lines.

From (3.2) and (3.3) we find that the $g_{\rho \pi \pi}$ at the two quark masses are consistent within the statistical error and also with the experiment $g_{\rho \pi \pi}=5.874 \pm 0.014$ given from the experimental results of the decay width $\Gamma_{\rho}=146.2 \pm 0.7 \mathrm{MeV}$ [18]. This suggests a weak quark mass dependence of the coupling constant. But our calculations are carried out only at the two quark masses, so calculations at more quark masses are necessary to obtain a definite conclusion for the quark mass dependence. We leave this issue to studies in the future.

In Fig. 3 we compare our results (PACS-CS) obtained in $2+1$ flavor QCD with those by ETMC [9, 10] and Lang et al. [11] in 2 flavor QCD. The left panel shows the effective coupling constant $g_{\rho \pi \pi}$ and the right panel displays the resonance mass $m_{\rho}$ as a function of $m_{\pi}^{2}$. A good 

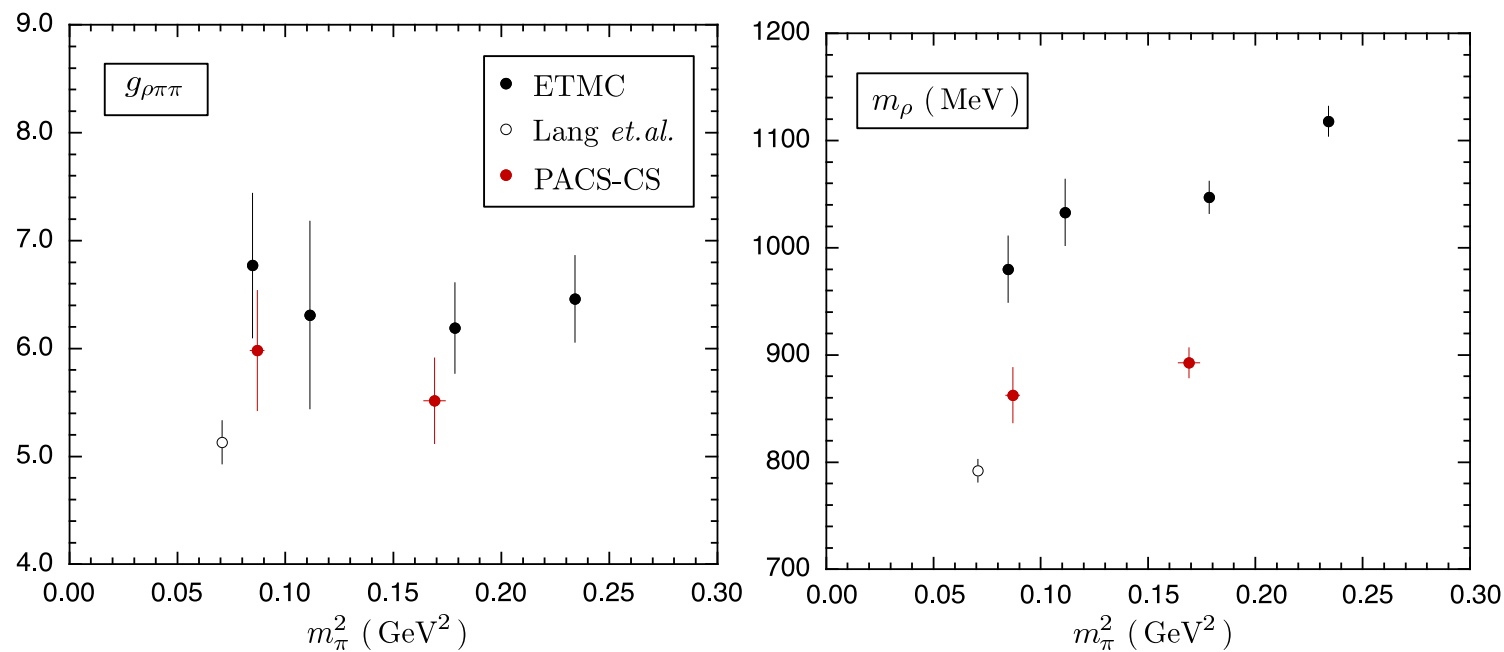

Figure 3: Comparison of our results (PACS-CS) obtained in $2+1$ flavor QCD with those by ETMC and Lang et al. in 2 flavor QCD. Left panel shows the effective coupling constant $g_{\rho \pi} \pi$ and right is the resonance mass $m_{\rho}$.

agreement between our result and ETMC is observed for $g_{\rho \pi \pi}$. The result for the coupling constant by Lang et al. takes a slightly smaller value, but it is almost consistent with other works.

We see, however, large discrepancy for the resonance mass $m_{\rho}$ in the right panel of Fig. 3. The three groups worked at a single lattice spacing, therefor a possible reason of the discrepancy is the discretization error due to the finite lattice spacing. We can also consider several other reasons, the reliability of the determination of the lattice spacing, the dynamical strange quark effect, the isospin breaking effect and so on, but a definite conclusion can not be given here.

\section{Summary}

We have reported on a calculation of the $P$-wave scattering phase shift for the isospin $I=1$ two-pion system and an estimation of the resonance parameters from the $N_{f}=2+1$ full QCD configurations with a large lattice volume. The calculations are carried out at two quark masses, which correspond to $m_{\pi}=410 \mathrm{MeV}$ and $300 \mathrm{MeV}$. Our results of the effective coupling constant $g_{\rho \pi \pi}$ at the two quark masses are consistent within the statistical error and also with the experiment. This suggests a weak quark mass dependence of the coupling constant. We find a discrepancy for the resonance mass $m_{\rho}$ among three lattice studies. Calculations near or on the physical point closer to the continuum limit are necessary for a precise determination of the resonance mass from lattice QCD. We leave this issue to studies in the future.

\section{Acknowledgments}

This work is supported in part by Grants-in-Aid of the Ministry of Education (Nos. 20340047, 20105001, 20105003, 20540248, 23340054, 21340049, 22244018, 20105002, 22105501, 22740138, 23540310, 22540265, 23105701, 10143538, 21105501, 23105708, 20105005 ). The numerical calculations have been carried out on PACS-CS at Center for Computational Sciences, University of Tsukuba. 


\section{References}

[1] S. Gottlieb, P.B. Mackenzie, H.B. Thacker and D. Weingarten, Phys. Lett. B134, 346 (1984).

[2] R.D. Loft and T.A. DeGrand, Phys. Rev. D39, 2692 (1989).

[3] UKQCD Collaboration, C. McNeile and C. Michael, Phys. Lett. B556, 177 (2003) [hep-lat/0212020].

[4] ETMC Collaboration, K. Jansen, C. McNeile, C. Michael and C. Urbach, Phys. Rev. D80, 054510 (2009) [arXiv:0906.4720 [hep-lat]].

[5] M. Lüscher, Commun. Math. Phys. 105, 153 (1986); Nucl. Phys. B354, 531 (1991).

[6] K. Rummukainen and S. Gottlieb, Nucl. Phys. B450, 397 (1995) [hep-lat/9503028].

[7] ETMC Collaboration, X. Feng, K. Jansen and D.B. Renner, PoS LATTICE2010, 104 (2010) [arXiv:1104.0058 [hep-lat]].

[8] CP-PACS Collaboration, S. Aoki et al., Phys. Rev. D76, 094506 (2007) [arXiv:0708.3705 [hep-lat]].

[9] ETMC Collaboration, X. Feng, K. Jansen and D.B. Renner, PoS LATTICE2009, 109 (2009) [arXiv:0910.4871 [hep-lat]].

[10] ETMC Collaboration, X. Feng, K. Jansen and D.B. Renner, Phys. Rev. D83, 094505 (2011) [arXiv:1011.5288 [hep-lat]].

[11] C.B. Lang, D. Mohler, S. Prelovsek and M. Vidmar, arXiv:1105.5636 [hep-lat].

[12] PACS-CS Collaboration, S. Aoki et al., Phys. Rev. D79, 034503 (2009) [arXiv:0807.1661 [hep-lat]].

[13] PACS-CS Collaboration, S. Aoki et al., arXiv:1106.5365 [he-lat].

[14] QCDSF Collaboration, M. Gockeler et al., PoS LATTICE2008, 136 (2008) [arXiv:0810.5337 [hep-lat]].

[15] BMW collaboration, J. Frison et al., PoS LATTICE2010, 139 (2010) [arXiv:1011.3413 [hep-lat]].

[16] PACS-CS Collaboration, S. Aoki et al., PoS LATTICE2010, 108 (2010) [arXiv:1011.1063 [hep-lat]].

[17] M. Lüscher and U. Wolff, Nucl. Phys. B339, 222 (1990).

[18] K. Nakamura et al. (Particle Data Group), J. Phys. G 37, 075021 (2010) 